\title{
Backside-Illuminated Silicon Photodiode Array for an Integrated Spectrometer
}

\author{
Tommy A. Kwa, Pasqualina M. Sarro, Member, IEEE, and Reinoud F. Wolffenbuttel
}

\begin{abstract}
A $\mathbf{p}^{+}-\mathrm{n}$, backside-illuminated photodiode array, to be used in an integrated silicon spectrometer, has been fabricated and characterized. For this type of application, illumination from the back is essential. The array is suspended in a nitride membrane for enhanced electrical and thermal isolation from the micromachined bulk. To prevent illumination from the front, the array is entirely covered with an aluminum layer, which also enhances the spectral responsivity. A model is described with which the performance of the backside-illuminated array was predicted. Furthermore, the performance of the photodiode array is compared to that of a conventional, front-illuminated array.
\end{abstract}

\section{INTRODUCTION}

$\mathbf{I}$ N CONVENTIONAL applications where an array of photodiodes is employed, the detectors are illuminated from the front. In this paper, a simple $\mathrm{p}^{+}-\mathrm{n}$ junction photodiode array is described, which is illuminated from the back. The array is applied in an integrated silicon spectrometer, as shown in Fig. 1, for the analysis of light in the visible spectrum. Besides a high responsivity in the spectral operating range, a low dark current is required. To achieve this, the photodiode array is made in a silicon membrane, which is suspended in a membrane of silicon nitride. Nitride is chosen because of its high mechanical strength, its compatibility with bipolar IC processing, and because it endures the etchant used to remove the bulk silicon. A top aluminum layer covers the entire array to prevent illumination from the front and also serves as a mirror to extend the absorption path. The resulting structure is shown in Fig. 2(f). The array consists of 12 photodiodes of $40 \times 1000 \mu \mathrm{m}^{2}$ each. A model is described with which the performance of the backside-illuminated array was predicted. The performance is compared with that of a similar frontilluminated array, and in Section V to measured data.

\section{DEVICE FABRICATION}

The fabrication process of the photodiode is given in Fig. 2. A (100), 2-5 $\Omega \cdot \mathrm{cm}$, p-type substrate with a $2.8-\mu \mathrm{m}$ thick $\mathrm{n}$ type epitaxial layer with a resistivity of $0.5 \Omega \cdot \mathrm{cm}$ is used. A

Manuscript received October 7, 1996. The review of this paper was arranged by Editor P. K. Bhattacharya.

T. A. Kwa was with the Laboratory for Electronic Instrumentation, Department of Electrical Engineering, Delft University of Technology, 2628 CD Delft, The Netherlands. He is now with Foxboro /ICT, San Jose, CA 95134 USA.

P. M. Sarro is with the Delft Institute of Microelectronics and Submicrontechnology (DIMES), 2600 GB Delft, The Netherlands.

R. F. Wolffenbuttel is with the Laboratory for Electronic Instrumentation, Department of Electrical Engineering, Delft University of Technology, 2628 CD Delft, The Netherlands.

Publisher Item Identifier S 0018-9383(97)02997-3.

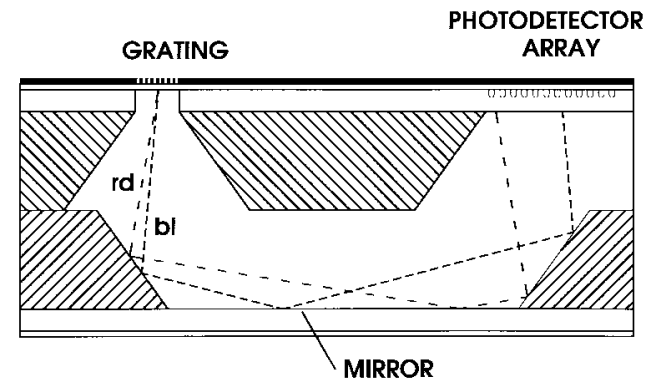

Fig. 1. Principle of the integrated silicon spectrometer.

deep p-type diffusion ( $8 \Omega / \square$ ) is performed to define channels that are to be etched away during the etching of the bulk. Subsequently, the cathodes of the photodiodes are formed with a deep n-type diffusion $(10 \Omega / \square)$, which is also used to contact the epilayer during the electrochemically controlled removal of the bulk silicon. Next, a thin oxide $(1200 \AA)$ is grown through which boron is implanted $\left(5.8 \cdot 10^{15} \mathrm{~cm}^{-2}, 150\right.$ $\mathrm{keV})$ to form the anodes of the photodiodes. A low tensilestress nitride layer [1] with a thickness of $4000 \AA$ is deposited by LPCVD [Fig. 2(a)]. This layer is patterned to form the nitride membranes [Fig. 2(b)]. Contact holes are etched and an aluminum layer is deposited $(0.6 \mu \mathrm{m})$ and patterned for the electrical interconnections [Fig. 2(c)]. A PECVD oxide $(0.8 \mu \mathrm{m})$ separates a second layer of aluminum $(0.6 \mu \mathrm{m})$. The oxide and nitride layers at the backside are stripped off [Fig. 2(d)] Finally, a 3500- $\AA$ thick PECVD nitride layer is deposited on the backside and patterned for the etch-mask [Fig. 2(e)]. Etching of the substrate takes place in a $\mathrm{KOH}$ solution with a concentration of 33 wt. $\%$ at a temperature of $90{ }^{\circ} \mathrm{C}$ using Electrochemically Controlled Etching (ECE) in a two-electrode configuration [2]. The etching stops at the epilayer, but continues where an isolation diffusion is present and eventually stops on the nitride layer [3] [Fig. 2(f)].

\section{Model to Determine the Spectral Response}

The spectral response of the photodiodes can be modeled using the wavelength-dependent absorption coefficient of silicon given in literature [4]. As the array is illuminated from the back, the p-n junction is rather far from the illuminated surface - compared to a front-illuminated array-and the generated charge carriers must travel a distance-dependent on the depth of absorption - through the epilayer to reach the junction. The ECE bulk-micromachining technique enables the fabrication of membranes sufficiently thin compared to the diffusion length of excess minority carriers to justify the 


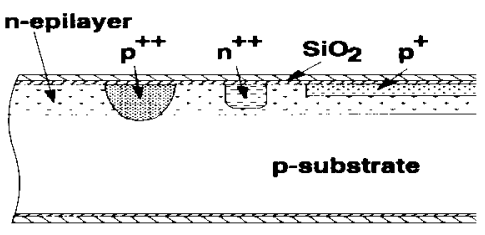

(a)

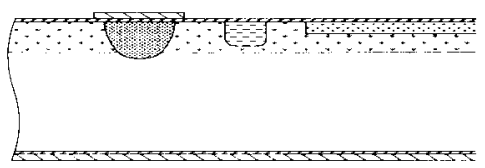

(b)

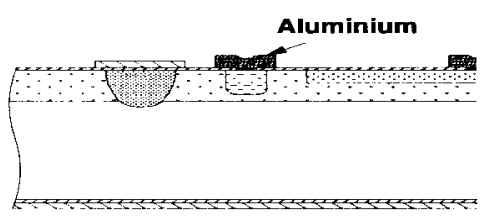

(c)

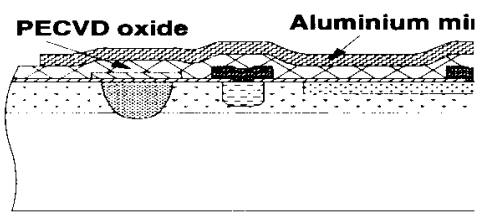

(d)

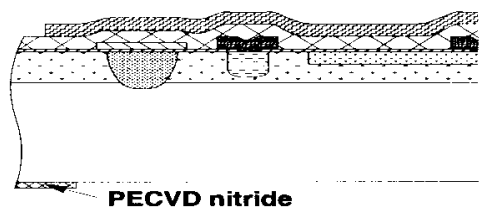

(e)

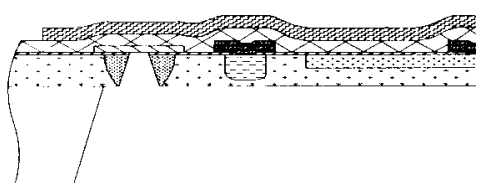

(f)

Fig. 2. Fabrication sequence of the suspended backside-illuminated photodiode array.

assumption that all carriers generated in the $2-4-\mu \mathrm{m}$ thick silicon membrane, and which are not lost due to recombination at the surface, contribute to the photocurrent of the diode. For carriers generated close to the surface however, this assumption cannot be made due to the presence of surface states. These are created at an abrupt ending of the crystal structure and form recombination centres [5].

The presence of surface states has especially an effect on the spectral response for blue light because of the high absorption at the surface at shorter wavelengths. The effect is less significant for longer wavelengths. For simplicity of modeling, the presence of surface states is accounted for by the introduction of a dead zone; i.e., a region in which all the generated carriers are considered to recombine at the surface and, thus, do not contribute to the photocurrent.

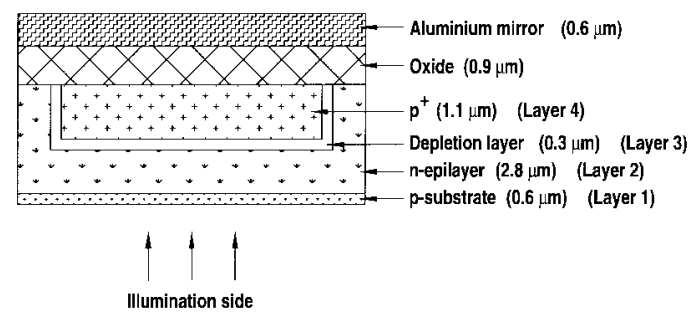

Fig. 3. Schematic of the backside-illuminated photodiode.

The photon flux, with wavelength $\lambda$, drops exponentially along the propagation path according to Beer's law

$$
\frac{F(\lambda, x)}{F_{0}(\lambda)}=e^{-\alpha(\lambda) x}
$$

where $F_{o}(\lambda)$ is the initial photon flux (just below the surface within the material), $F(\lambda, x)$ the remaining flux deeper into the silicon at a depth $x$ in the material, and $\alpha(\lambda)$ the wavelengthdependent absorption coefficient of the material. Equation (1) is derived from Poisson statistics according to which the absorption probability of photons with wavelength $\lambda$, at depth $x$, is

$$
P(\lambda, x)=\alpha(\lambda) e^{-\alpha(\lambda) x} .
$$

The number of photons, with wavelength $\lambda$, absorbed in a layer from a depth $d$ to $L$ is equal to the product of the initial photon flux and the total absorption ratio of photons in the layer, thus

$$
N(\lambda)=F_{0}(\lambda) \int_{d}^{L} \alpha(\lambda) e^{-\alpha(\lambda) x} d x .
$$

The responsivity for monochromatic light is defined as the ratio of the photocurrent, $J_{\mathrm{ph}}$, to the optical power, $P_{\mathrm{opt}}$

$$
S(\lambda)=\frac{J_{\mathrm{ph}}(\lambda)}{P_{\mathrm{opt}}(\lambda)} .
$$

The photocurrent density is proportional to the number of generated carriers $\eta \cdot N(\lambda)$,

$$
J_{\mathrm{ph}}(\lambda)=\eta N(\lambda) q,
$$

where $\eta$ is the internal quantum efficiency. The optical power of a photon flux is inversely proportional to its wavelength

$$
P_{\text {opt }}(\lambda)=F_{0}(\lambda) \frac{h c}{\lambda} .
$$

Substituting (5) and (6) into (4) yields a responsivity proportional to the product of the total photon absorption ratio and the wavelength,

$$
S(\lambda)=\frac{\eta \lambda q}{h c} \int_{d}^{L} \alpha(\lambda) e^{-\alpha(\lambda) x} d x .
$$

With the schematic shown in Fig. 3, the contribution of each layer to the photocurrent can be calculated to obtain the expected spectral responsivity. As a first wavelengthdependent effect, part of the photon flux incident on the silicon reflects on the surface. The part that penetrates into the silicon is, at normal incidence, $1-R_{S i}(\lambda)$, where $R_{S i}(\lambda)$ is the reflectance at the surface. In the following a value of $33 \%$ is chosen for $R_{S i}(\lambda)$ [4]. 


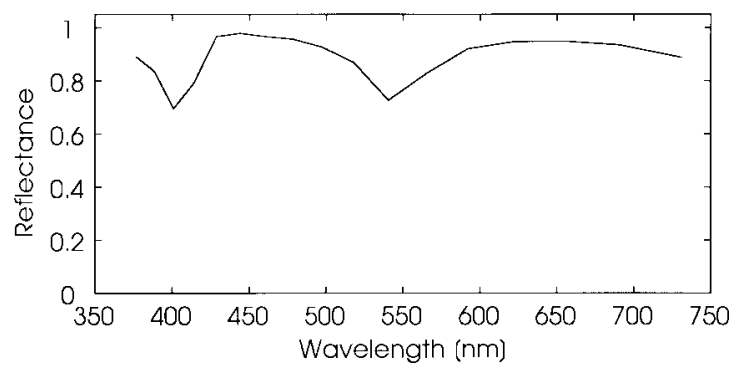

Fig. 4. Reflectance of an $\mathrm{SiO}_{2} / \mathrm{Al}$-layer on silicon as a function of the wavelength. The thickness of the oxide is $0.9 \mu \mathrm{m}$.

Transmitted photons enter the silicon that is divided into layers 1 to 4 . During the removal of the bulk silicon, the ECEstop was used. In this technique, the etching stops on a biased silicon layer that forms the membrane. For this purpose, the epitaxial layer was employed. The epilayer-substrate junction is reverse biased at a voltage of $1.5 \mathrm{~V}$. This results, for the doping levels used, in a depletion layer that extends about $0.6 \mu \mathrm{m}$ into the substrate. Etching stops when the etching front reaches this depletion layer [6], and therefore, a thin substrate layer is expected to remain under the epilayer. The contribution of charge carriers generated in this layer to the photocurrent is negligible and consequently, the influence of this layer on the spectral response can conveniently be included in the thickness of the dead zone (Layer 1).

In the epilayer an internal quantum efficiency equal to unity is assumed, which is valid within the part of the spectrum being considered [7]. It is assumed that in the depletion layer (Layer 3) all the generated charge carriers contribute to the photocurrent, and that half of all the charge carriers generated in the undepleted parts of the epilayer (Layers 2 and 4) diffuses to the $\mathrm{p}^{+}-\mathrm{n}$ junction and contributes to the photocurrent (the other half recombines at the surface).

The oxide-aluminum mirror reflects the remaining photon flux back into the silicon, resulting in a doubling of the effective absorption path. The reflectance, $R_{\text {Mirror }}(\lambda)$, of the $\mathrm{SiO}_{2} /$ Al-layers on top of the silicon surface can be calculated as a function of wavelength, and the plot is given in Fig. 4 (Layers 4, 3, and 2). The mirror is only effective at wavelengths with a penetration depth larger than $4.8 \mu \mathrm{m}(\lambda=600$ $\mathrm{nm}$ ), so the dips at lower wavelengths can be disregarded. Summing the contribution of each layer, we obtain

$$
\begin{aligned}
N(\lambda)= & F_{0}(\lambda)\left(1-R_{S i}(\lambda)\right)\left(\sum_{i=2}^{4} c_{i} \int_{\text {Layer }_{i}} \alpha(\lambda) e^{-\alpha(\lambda) x} d x\right. \\
& \left.+R_{\text {Mirror }}(\lambda) \sum_{i=4}^{2} c_{i} \int_{\text {Layer }_{i}} \alpha(\lambda) e^{-\alpha(\lambda) x} d x\right)
\end{aligned}
$$

where $c_{i}=1 / 2$ for $i=2,4$ and $c_{i}=1$ for $i=3$.

Substituting (5), (6), and (8) into (4), and assuming a dead zone of $0.6 \mu \mathrm{m}$, the expected spectral responsivity is found as shown in Fig. 5(f). In the blue region the device has a low response, which increases with increasing wavelength. The response has a peak at $\lambda=650 \mathrm{~nm}$. The influence of a substrate layer is also shown in Fig. 5 for various thicknesses of the dead zone.

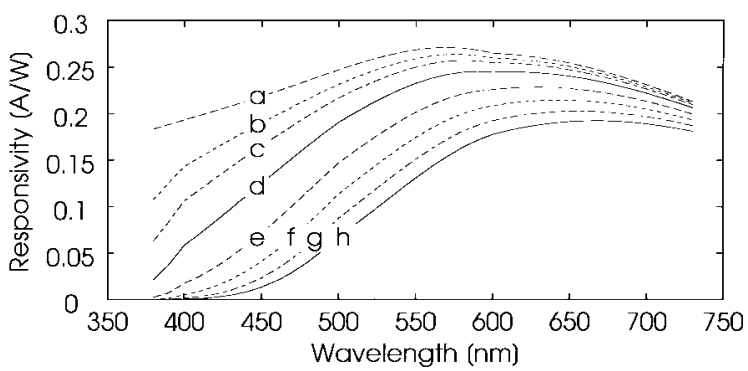

Fig. 5. Calculated spectral responsivity for various thicknesses of the dead zone: (a) $0 \backslash$; $\mu \mathrm{m}$; (b) $0.05 \mu \mathrm{m}$; (c) $0.1 \mu \mathrm{m}$; (d) $0.2 \mu \mathrm{m}$; (e) $0.4 \mu \mathrm{m}$; (f) $0.6 \mu \mathrm{m} ;$ (g) $0.8 \mu \mathrm{m}$; (h) $1.0 \mu \mathrm{m}$.

\section{Device Measurement Method}

The diode electrical characteristics and optical responsivity were measured using an HP4142B DC Source/Monitor, controlled by an HP9000/700 computer. A $100 \mathrm{~W}$ quartz tungsten halogen lamp (Oriel 6333) with a 1/8 m monochromator (Oriel 77250) with a ruled grating (Oriel 77298) with an optimum wavelength region between 330 and $750 \mathrm{~nm}$, was used as the light source. The light was coupled into the photodiode through a glass fiber optic bundle (Oriel 77525). A variable slit (Oriel 77263) was adjusted to pass a spectral bandwidth of $4 \mathrm{~nm}$. The spectral responses (wavelength range 380-730 $\mathrm{nm}$ ) were measured at several bias voltages. The backside-illuminated photodiode arrays were mounted in a specially prepared IC housing to permit illumination from the back while enabling wire-bonding at the front. The optical system was calibrated by measuring the response of a calibrated Si reference photodiode (Oriel 7180) to a wavelength scan. The responsivity of the photodiodes was then determined by replacing the reference diode and repeating the wavelength scan.

\section{EXPERIMENTAL RESULTS AND DISCUSSION}

\section{A. Dark Current}

As a reference to the backside-illuminated photodiodes, the reverse current-voltage characteristic of a nonmicromachined, front-illuminated photodiode with identical layout and doping concentrations was measured, and the result is shown in Fig. 6. Up to the breakdown voltage of about $30 \mathrm{~V}$, the dark current increases to about $1 \mathrm{nA}$. The higher dark current of the micromachined backside photodiode is due to surface defects and can be reduced by thermal treatment. However such a step is poorly compatible with the integrated circuits.

\section{B. Spectral Response}

Fig. 7 presents the spectral response of the front-illuminated photodiodes at zero bias and reverse-biased at $2.5 \mathrm{~V}$ with reverse-biasing of the epilayer-substrate junction as well. Increasing the reverse bias from 0 to $2.5 \mathrm{~V}$ results in a small increase of the spectral response, up to a maximum of $12 \%$. As the increase in signal level does not outweigh the increase of the dark current, the reverse voltage was restricted to 2.5 $\mathrm{V}$. The two peaks in the responses (at $\lambda=470$ and 560 $\mathrm{nm}$ ) originate from the passivation oxide layer, which acts as an optical interference filter. Silicon has a low absorption 


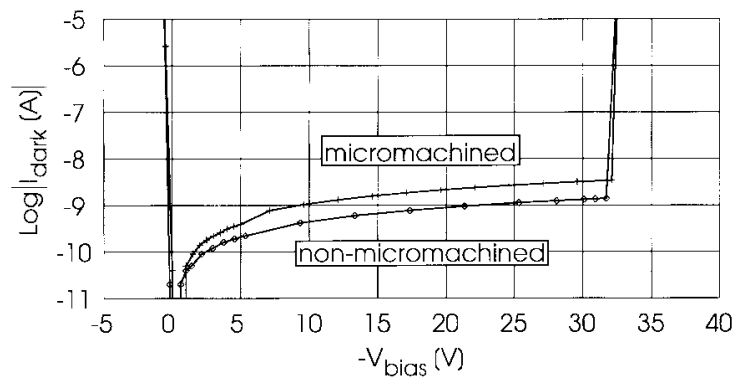

Fig. 6. Reverse current-voltage characteristics of a nonmicromachined and a micromachined photodiode.

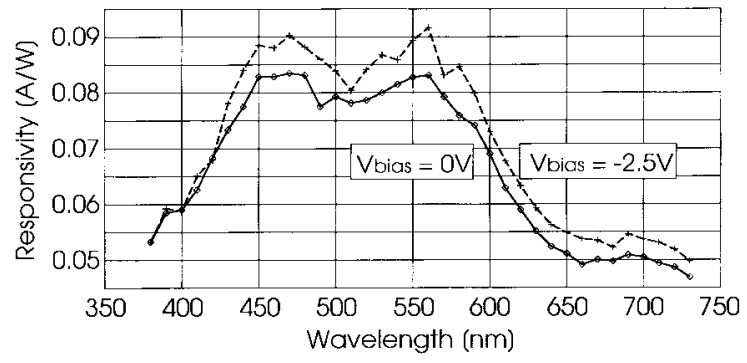

Fig. 7. Spectral response of a front-illuminated photodiode at zero bias and reverse-biased at $2.5 \mathrm{~V}$.

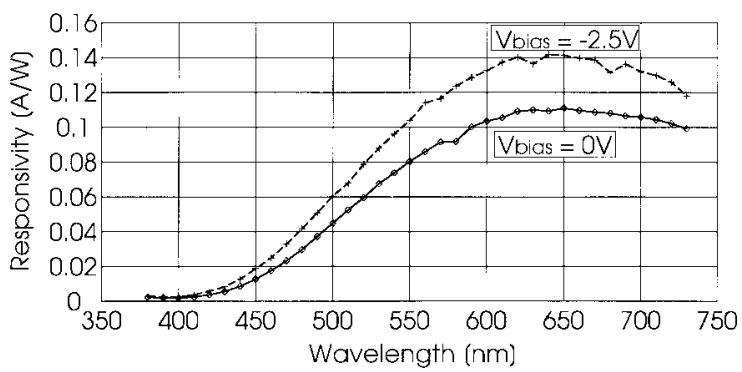

Fig. 8. Spectral response of a backside-illuminated photodiode reverse-biased at 0 and $2.5 \mathrm{~V}$.

coefficient for red light which can therefore penetrate deeply into the silicon. Carriers generated beyond the biased episubstrate junction (thus, originating mainly from red light) do not reach the $\mathrm{p}^{+}$-epilayer junction, as these are already collected in the epi-substrate junction. As a result, the response falls off for the longer wavelengths.

The spectral response of the backside-illuminated photodiodes reverse-biased at 0 and $2.5 \mathrm{~V}$ is shown in Fig. 8. In contrast to the front-illuminated diodes, the response is much lower for the shorter than for the longer wavelengths, which is because a) blue light is absorbed very shallowly; i.e., at the etched surface, where the recombination velocity is high due to the large number of surface states at the abrupt crystal ending [5], and b) carriers generated in the remaining substrate layer are collected in the epi-substrate junction. Red light, in contrast, can penetrate much deeper into the silicon (thus, beyond the epi-substrate junction) and consequently the response for the longer wavelengths is much higher. The response has a peak at $\lambda \approx 650 \mathrm{~nm}$ as was already expected from the calculations given in Section III and closely matches the curve for a dead zone of $0.6-1.0 \mu \mathrm{m}$.

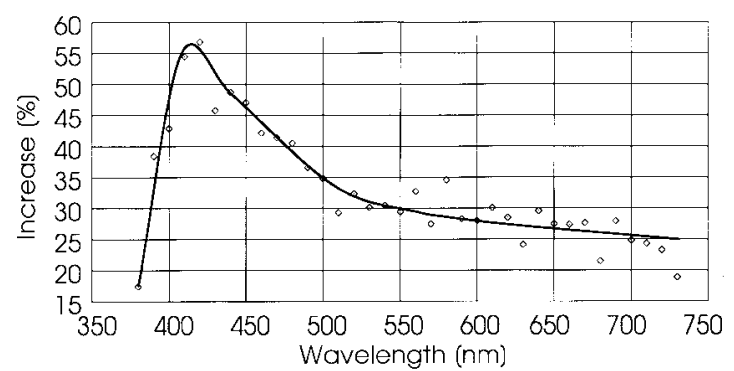

Fig. 9. Relative increase of the spectral response of the backside-illuminated photodiode when increasing the reverse-bias voltage from 0 to $2.5 \mathrm{~V}$.

This is in agreement with the measured thickness of the remaining substrate under the epilayer. The absolute values of the response are in close agreement with the expected ones from the calculations, justifying the assumption made that half of all the charge carriers generated in the undepleted parts of the epilayer contributes to the photocurrent. Increasing the bias voltage results in an increase of the response due to the increasing depletion layer. Moreover, the increase in the short-wavelength region is relatively larger than in the longwavelength region (see Fig. 9). For the shortest wavelengths $(380-390 \mathrm{~nm})$, however, the increase is smaller due to the high absorption and high recombination rate at the surface.

\section{CONCLUSIONS}

Using standard bipolar IC processing and a single bulkmicromachining etch-step, a suspended backside-illuminated silicon photodiode array has been realized. The spectral responsivity has a peak value of $0.14 \mathrm{~A} / \mathrm{W}$ at a wavelength of $650 \mathrm{~nm}$. In contrast, the front-illuminated array has a peak responsivity of $0.09 \mathrm{~A} / \mathrm{W}$ at 470 and $560 \mathrm{~nm}$. Despite the removal of the bulk silicon, which introduces surface states at the etched photodiode surface, the dark current is not increased very much compared to that of the front-illuminated array. The dark current is less than $0.5 \mathrm{nA}$ at reverse bias voltages below $2.5 \mathrm{~V}$. The array is suspended in a nitride membrane for electrical and thermal isolation. As the photodiodes are fabricated in a standard bipolar process, compatibility with electronic circuitry processing is maintained.

\section{ACKNOWLEDGMENT}

The authors would like to thank the staff of the Delft Institute of Microelectronics and Submicrontechnology (DIMES), in particular W. van der Vlist, for processing the devices. The authors also acknowledge P. J. French for discussions on fabrication, and the assistance of R. P. van Kampen with the measurements and fruitful discussions of the results with S. R. in 't Hout.

\section{REFERENCES}

[1] P. J. French, P. M. Sarro, R. Malleé, and R. F. Wolffenbuttel, “Optimization of a low-stress silicon nitride process for surface micromachining applications," Abstr. Eurosensors VII, Toulouse, France, Sept. 25-28, 1994, p. 205.

[2] P. M. Sarro and A. W. van Herwaarden, "Silicon cantilever beams fabricated by electrochemically controlled etching for sensor applications," J. Electrochem. Soc., vol. 133, 1986, pp. 1724-1729. 
[3] T. A. Kwa and R. F. Wolffenbuttel, "Integrated grating/detector array fabricated in silicon using micromachining techniques," Sensors and Actuators A, vol. 31, 1992, pp. 259-266.

[4] D. E. Aspnes, "Optical functions of intrinsic silicon: table of refractive index, extinction coefficient and absorption coefficient versus energy," in Properties of Silicon. London, U.K.: INSPEC, 1988, pp. 72-79.

[5] A. S. Grove, Physics and Technology of Semiconductor Devices. New York: Wiley, 1967, ch. 5.

[6] R. Huster, A. Kovács, and A. Stoffel, "Etch-stop behavior of depletion layers," in Proc. IEEE Workshop on Micro-Electro Mechanical Systems, Travemünde, Germany, Feb. 4-7, 1992, pp. 79-82.

[7] O. Christensen, "Quantum efficiency of the internal photoelectric effect in silicon and germanium," J. Appl. Phys., vol. 47, 1976, pp. 689-695.

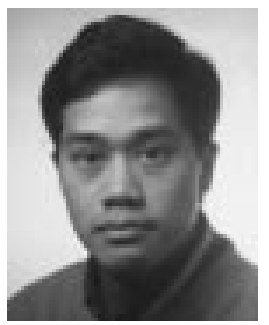

Tommy A. Kwa received the M.Sc. and Ph.D. degrees in electrical engineering from Delft University of Technology, Delft, The Netherlands, in 1990 and 1995, respectively, where he was performing research on the application of silicon micromachining techniques for an integrated spectrometer.

In 1995, he joined Foxboro/ICT, Inc., San Jose, $\mathrm{CA}$, where he has been working on the design and fabrication of silicon pressure sensors.

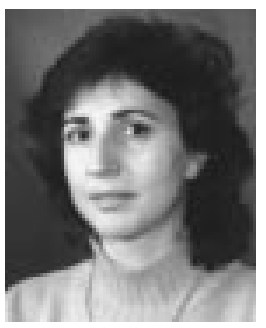

Pasqualina M. Sarro (M'83-S'84-M'87) received the Laurea degree in solid-states physics from the University of Naples, Italy, in 1980. From 1981 to 1983 , she was a post-doctoral fellow in the Photovoltaic Research Group of the Division of Engineering, Brown University, Providence, RI. In 1987, she received the Ph.D. degree in electrical engineering from Delft University of Technology, Delft, The Netherlands.

Since 1987, she has been with the Delft Institute of Microelectronics and Submicron Technology (DIMES), Delft University, where she is responsible for research on integrated silicon sensors and microsystems technology.

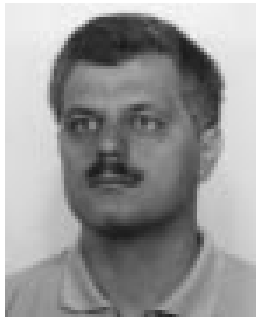

Reinoud F. Wolffenbuttel received the M.Sc. degree in 1984 and the Ph.D. degree in 1988, both from the Delft University of Technology, Delft, The Netherlands. His thesis work dealt with the application of silicon to color sensing.

Since 1986, he has been with the Laboratory of Electronic Instrumentation of the Delft University of Technology; from 1986 to 1993 as an Assistant Professor, and since 1993, as an Associate Professor $\mathrm{He}$ is involved in instrumentation and measurement in general and on-chip functional integration of microelectronic circuits and silicon sensor, fabrication compatibility issues, and micromachining in silicon and microsystems in particular. In 1992, he was a Visiting Scientist at the University of Michigan, Ann Arbor, where he was involved in research on low-temperature wafer-to-wafer bonding. 